\title{
Cases of Adverse Drug Events in Pediatrics: Prospective Study at a Hospital in Rabat (Morocco)
}

\author{
Laila Torissi ${ }^{1,2, *}$, A. Soulaymani ${ }^{1}$, A. Mokhtari ${ }^{1}$, Soulaymani Rachida ${ }^{3}$ \\ 'Laboratoire de Génétique et Biométrie, Faculté des Sciences, Université Ibn Tofail, Kénitra, MOROCco. \\ 2Direction du Médicament et de la Pharmacie (DMP), Ministère de la Santé, Rabat, MOROCCO. \\ ${ }^{3}$ Antipoison Center and Pharmacovigilance of Morocco, Rabat, MOROCCO.
}

\begin{abstract}
Pharmacovigilance aims to guarantee the safe use of medicines. It is based on the reporting of adverse effects by health professionals, industry and the public. Pharmacovigilance is the monitoring of drugs and the prevention of the risk of adverse effects resulting from their use, whether this risk is potential or proven. It relies on the spontaneous reporting of adverse events by health professionals and patients, the collection of adverse effects for the detection of signals the establishment of preventive measures. Many studies have highlighted the importance of harm related to Adverse Drug Events especially in children. In connection with this, we carried out a study of the undesirable effects of medicines at the Children's Hospital of Rabat. This study was authorized by the Ministry of Health and
\end{abstract}

lasted 6 months during which were recollected several cases including 10 cases presented in this article.

Key words: Newborns, Children, Adverse drug reactions, Pharmacovigilance, Prospective study.

\section{Correspondence}

Laila Torissi, Laboratoire de Génétique et Biométrie, Faculté des Sciences, Université Ibn Tofail, Kénitra, MOROCCO.

Phone: 00212-619033641

Email: torissileila@yahoo.fr

DOI: 10.5530/jyp.2018.10.80

\section{INTRODUCTION}

In view of the under declaration of adverse drug reactions (ADRs) there is a need for optimizing their reporting.Among the objectives is to study the cases of Adverse Drug Reactions (ADRs) and describe them and also to study the drugs involved in pediatrics. It's a study who makes health professionals aware of the importance of the notification to avoid serious consequences on the health of the pediatric population. Doctors are often required to prescribe medicines designed for adults which can have even dramatic consequences. The objective also was to propose preventive strategies and encourage regulatory authorities to carry out corrective actions.

\section{MATERIALS AND METHODS}

A prospective observational study about Adverse Drug Reactions in children was conducted for 6 months at Children's Hospital of Rabat (HER) between February and June 2013. The population included in this study was hospitalized patients in the various pediatric services of the hospital having an adverse drug event which was the subject to consultation or hospitalization. Inclusion criteria were Patients who had an adverse drug event that had been referred for consultation, hospitalization, prolonged hospitalization or occurring during hospitalization. The study was authorized by the Ministry of Health and has the agreement of the hospital's ethics committee.

The registers with the notice cards of the ADRs were deposited in the departments of the Hospital, the hospital pharmacy was sensitized when with the study and also the Hospital staff (Doctors, Pharmacists, Nurses). Once there were reports of ADRs, the completed forms with the data were forwarded to the Poison Control and Pharmacovigilance Center (Centre Antipoison et de Pharmacovigilance) where the case account- ability study and the cause-effect relationship study were conducted by the experts of the Center. The result of the study of the causality and the cause-and-effect relationship of drugs implicated or not in the occurrence of the adverse effect was sent to notifiers at the hospital level; the cards ("Fiches de Notifications d'effets indésirables") were not always complete; there could be a lack of important data to carry out the accountability study. The cases (Table 1) were presented as they are and this is a real description of the difficulties that can be encountered when studying cases in the context of pharmacovigilance.

\section{Accountability}

Accountability or cause-and-effect relationship between event clinical or biological and taken drug was performed according to the French method. ${ }^{1}$ Reference method used by the Moroccan Center pharmacovigilance, it is based on a on the chronological criteria of the event in relation to taking the drug, its judgement, and its readministration and, on the other hand, it relies on semeiological criteria in trying to find a pharmacological explanation at the event, supporting diagnoses differential knowing that the adverse effect medicated is a diagnosis disposal. A score is awarded to each observation to rate the degree of responsibility of the drug, in questionable, plausible, probable accountability, or very likely. The accountability session ends with Bibliographic confrontation necessary and indispensable for the analysis of any case of pharmacovigilance.

Intrinsic accountability (imputabilité intrinsèque) relies on seven criteria divided into two groups: chronological criteria and semiological criteria. The combination of chronological and semiological criteria makes it possible to determine the intrinsic accountability score I: 
- I4: very likely accountability

- I3: probable accountability

- I2: plausible accountability

- I1: doubtful imputability

- I0: Incompatible Accountability

Extrinsic accountability: these scores are as follows and relate to the references mentioned:

B3: Notorious effect:

- Drug Dictionary

- Vidal, Martindale

- Meyler's Side Effect

B2: Notorious effect in the usual documents, published 1 or 2 times with a relatively different physiology or reported with a neighboring drug.

B1: Effect not described according to B3 or B2 definitions in the works namely to qualify B3.

B0: Effect appearing completely new after a search comprehensive.

\section{RESULTS}

\section{Case 1: Case of a maculopapular rash under intravenous Ampicillin}

\section{Information about the drug administered}

This medicine is an antibiotic of the beta-lactam family of the penicillin group. It is indicated for the treatment of bacterial infections with sensitive germs.

The patient named «fille de Fatima» 7 days old, $(3,400 \mathrm{~kg}$ ) (female), gestational age AT. Her history and clinical field were: suspicion of MFI (Maternal-Fetal Infection), respiratory distress, perinatal asphyxia II. Same rash noted especially during breastfeeding (mother under Amoxicillin). The date of onset was 21/04/2013, the conduct adopted was the discontinuation of the drug. The evolution of the patient was favorable. The dosage and route of administration: $100 \mathrm{mg} / \mathrm{kg} /$ day IV. Start date: $26 / 04 / 2013$ and stop date: $27 / 04 / 2013$. The accountability of the case carried out into the Poison Control and Pharmacovigilance center of Rabat (CAPM) using the French method revealed that: The time of onset of the adverse reaction is compatible, the evolution of the adverse events is suggestive because regression stopped, the semiology is questionable because the differential diagnoses have not been eliminated and other non-pharmacological explanations are possible. On the other hand, the data of the literature allowed us to say that the penicillins are providers of this type of lesions.

Case 2: Case of a generalized eruption, marbling and cyanosis under SG 10\% (Serum Glucose (SG IV 10\%) and Electrolyte $\mathrm{Na}^{+}, \mathrm{K}^{+}, \mathrm{Ca}$ (Sodium, Potassium, Calcium) indicated for parenteral nutrition

The patient called "Fils de Fatima" (male), 7 h old, $1.500 \mathrm{Kg}$ gestational AT, his history and clinical field was: intrauterine growth retardation. The date of onset was 21/05/2013 and the delay appears after taking was a few seconds. The differential diagnosis was gas embolism and the conducted adopted was the discontinuation of the drug and reduction of the dose. The corrective treatment was the injection of Saline Solution $0.9 \%$ and oxygen therapy. The evolution of the event was favorable.

The accountability of the case carried out into the Poison Control and Pharmacovigilance center of Rabat (CAPM) using the French method revealed that the attribution of the adverse effects to SG IV 10\% according to the French method revealed a doubtful cause-and-effect relationship because the time of onset of the adverse reaction is inconsistent, the evolution of the adverse event is suggestive because regression stopped, the semiology is doubtful because other non-pharmacological explanations are possible. In addition, data from the literature did not allow us to say that SG IV 10\% can be responsible for this type of adverse effects.

\section{Case 3: Case of Myelosuppression under the association drug of Trimethoprim and Sulfamethoxazole}

The myelosuppression occurred 3 days after administration of the association drug of Trimethoprim and Sulfamethoxazole to an infant; (case notified by a doctor of P3 Depatment of the Children's Hospital)

The intrinsic imputability of the case, according to the French method, revealed that the cause-and-effect relationship between taking this drug and the appearance of bone marrow suppression is "I2". Indeed, the evolution of the adverse effect is "inconclusive" since it is not mentioned on the declaration form. In addition, the semiology is "S1" since it is not suggestive of the role of drugs (not related to a pharmacological effect of the drugs administered) and other non-drug explanations have not been eliminated (differential diagnoses). Bibliographically, bone marrow suppression is an adverse effect rarely described when prescribing an antibacterial sulphonamide, unlike other haematological toxicities (neutropenia, thrombocytopenia, haemolytic anemia). These cases of medullary aplasia seem to be preferentially related to an immunoallergic mechanism.

According to the summary of product characteristics of the association Trimethoprim and Sulfamethoxazole (update on 17/06/2017) from the database of "The Agence Nationale de sécurité du Médicament et des produits de santé (ANSM)" there are adverse reactions hematological and lymphatic system disorders like: Leukopenia, Granulocytopenia, Thrombocytopenia, Anemia (megaloblastic, haemolytic / autoimmune, aplastic) (rare) and Methaemoglobinemia, Agranulocytosis, Pancytopenia (very rare).

Case 4: Case of biological hepatitis under Valproic acid, phenobarbital, Piracetam and paracetamol

The patient named "B.B" (male), 26 months old. The results of transaminase analyzes were (ASAT: 1250 UI and ALAT: 2513 UI) with cholestasis. The Drugs taken were Valproic acid, phenobarbital, Piracetam and paracetamol (oral).

The accountability of the case using the French method revealed that According to the data of the literature, hepatic damage under phenobarbital of severe and sometimes fatal evolution has been reported exceptionally. The mortality risk is estimated at $1 / 10,000$ for the general population and 1/500 for high-risk pediatric patients, including those under 3 years of age.

In the vast majority of cases, liver injury with phenobarbital occurs during the first 6 months of treatment, usually between the $2^{\text {nd }}$ and $12^{\text {th }}$ week. This toxicity is probably related to a metabolite of valproic acid that has a direct toxicity to liver cells in vitro. This risk is significantly increased when valproic acid is prescribed in antiepileptic combination therapy (1/7000 tale 1/500) probably by inducing effect of the CYP 450 enzyme. The taking of Phenobarbital, known for its inductive effect, by this patient could be a potential risk factor.

Regarding the hepatotoxicity of paracetamol, liver damage observed is toxic. The first manifestations, mainly an elevation of ASAT and ALAT aminotransferases $(>100 \mathrm{xN})$, occur between 24 and $48 \mathrm{~h}$ after ingestion. ASAT activity is often more important than ALAT activity. These liver disorders are related to the increased production of reactive metabolites by CYP 450 . Most often, this results from the ingestion of a massive dose of paracetamol, more rarely, at therapeutic doses.

In this observation, the study of accountability has been limited by the lack of several informations: Time to onset of liver injury, The periodicity of liver function monitoring during the first 6 months of treatment, The 
Table 1: Cases of adverse drug reactions (ADRs).

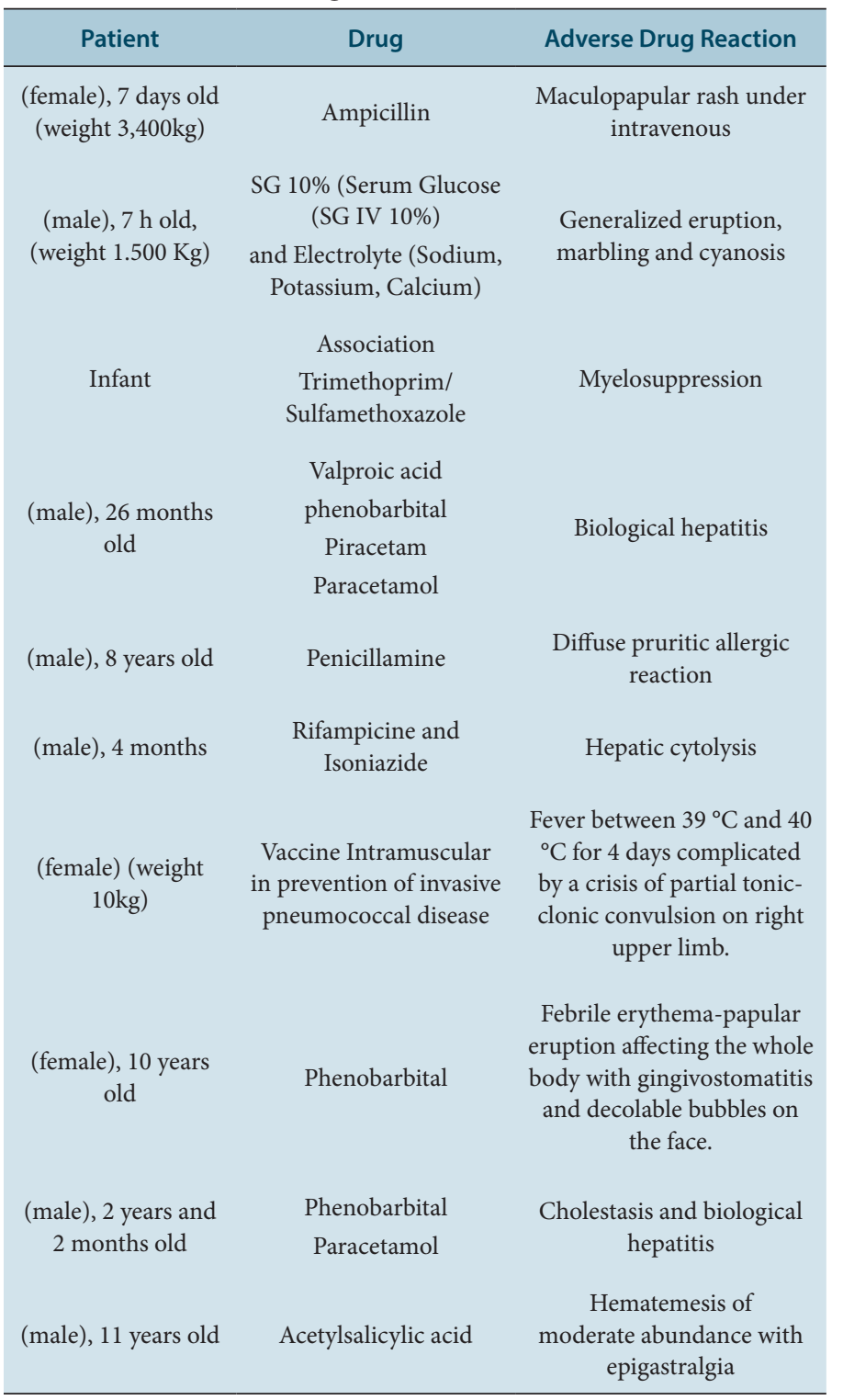

level of transaminases before the start of treatment, Other differential diagnoses evoked other hepatitis A, The conduct adopted by the treating physician.

\section{Case 5: Diffuse pruritic allergic reaction}

Patient called «xx», 8 years old (male). The adverse reaction was observed after taking the first dose of Penicillamine $300 \mathrm{mg}$ indicated for Wilson disease. The date of onset was 14/05/2013. The dosage and route of administration was $1 / 4 \times 2 /$ day (a quarter of a tablet twice a day) (progressive administration).

The accountability of the case carried out into the Poison Control and Pharmacovigilance center of Rabat (CAPM) using the French method revealed that according to data from the literature, penicillamine is responsible for early cutaneomucous rash and pruritus and other lateonset gingivitis, stomatitis, aphtoid lesion, toxidermy, pemphigus.

Cross-allergies between betalactamines and D-penicillamine are possible in some individuals.
For the accountability of this pharmacovigilance, essential parameters for the analysis are missing:

The delay of appearance of the undesirable effect: in hours days or months, the corrective treatment yes or no, the evolution of the event, if the drug stops or the adverse effect reappears after reintroduction.

\section{Other cases observed with $A D R(s)$ and notified were}

\section{Case 6: Hepatic cytolysis under Rifampicine and Isoniazide (Date of} notification: 15/04/2013):

The patient Y. L (male), 4 months old with clinical background with Oral thrush "Mugûet" resistant. The description of clinical and paraclinical adverse effect was Hepatic cytolysis.

The date of onset was 29/03/2013 and the differential diagnosis eliminated was the high hepatic transaminases (ASAT).Taking Rifampicine and Isoniazide started on 21/03/2013; The stopping date of Rifampicine and Isoniazide was $01 / 04 / 2013$. The evolution of the patient was favorable.

In addition to digestive disorders, the main adverse effect of RIFAMPICIN is hepatotoxicity. Hyperbilirubinemia (by inhibition of its secretion) and an increase in rapidly occurring liver enzymes after initiation of treatment are observed in $4 \%$ of patients; hepatitis is found in $3 \%{ }^{2}$ RIFAMPICIN potentiates hepatic toxicity of ISONIAZID ${ }^{3-4}$ probably by its effect on inducers of ISONIAZID metabolism, leading to increased production of its toxic metabolites. ISONIAZID is one of the most toxic anti-tuberculosis drugs for the liver. The increase in transaminases is observed in 10 to $25 \%$ of cases.

Case 7: Fever between $39^{\circ} \mathrm{C}$ and $40^{\circ} \mathrm{C}$ for 4 days complicated by a crisis of partial tonic-clonic convulsion on right upper limb with Vaccine: (Case notified by Pediatric Nephrology Department on 12/04/2013)

The Patient named I.X (female) (weight $10 \mathrm{~kg}$ ). The drug tacked was the vaccine Intramuscular in prevention of invasive pneumococcal disease (vaccine polyoside pneumococcique sérotype 1 conjugué à la protéine vectrice CRM197 $\rightarrow$ 2, 2 microgrammes de polyoside pneumococcique) .The Date of appearance on 08/04/2013 and the delay of appearance after taking $8 \mathrm{~h}$. The treatment carried out was to put the patient under Phenobarbital. The differential diagnosis eliminated was by EEG: posterior temporal irritative foci. Subject not yet reinstated.

The most commonly reported adverse reactions in children aged 6 weeks to 5 years reactions at the vaccination site, fever and irritability, loss of appetite and hypersomnia and / or decreased sleep.

The Nervous system disorders: Uncommon: Seizures (including febrile seizures) Rare: episode of hypotonia-hyporesponsiveness.

\section{Case 8: Febrile erythema-papular eruption affecting the whole body} with gingivostomatitis and decolable bubbles on the face (Case notified by Pediatric Nephrology Department on 02/04/2013):

The patient R. S (female), 10 years old, $24 \mathrm{~kg}$ weight was operated for astrocytoma of the posterior Cerebral Face on 14/03/2013 and put under Phenobarbital 2 tablets / day orally indicated us anti-comity prophylaxis. The starting date for taking the drug was 05/03/2013 and it was stopped on 30/03/2013. The description of clinical and para-clinical ADRs: febrile erythema-papular eruption affecting the whole body with gingivostomatitis and decolable bubbles on the face. The date of onset of the ADRs on 30/03/2013.The conducted adopted was the discontinuation of the drug and hospitalization. The patient was not yet reinstated and followed in dermatology.

Phenobarbital is one of the drugs strongly associated with Stevens-Johnson Syndrome: The mucous membrane of the mouth and the edge of the lips is almost always reached. The skin lesions are erythematous confluent macules with a purpuric center. Evolution of lesions: Formation of vesicles and bubbles, the zone development with a detachment of the epidermis. ${ }^{5}$ 


\section{Case 9: Cholestasis and biological hepatitis (notified on 3/05/2013):}

The patient (B.B) 2 years and 2 months (male). Patient with epilepsy under Phenobarbital 35mg/kg /day for 6 months and Paracetamol 3\% (syrup) for 3 days for fever. The differential diagnosis was viral hepatitis A. The description of clinical and para-clinical ADRs was cholestasis and biological hepatitis (asymptomatic) (with hepatic transaminase levels ASAT: 1250 IU and ALT: 2513 IU)

The frequent hepatobiliary disorders by Phenobarbital are isolated increase of gamma glutamyltranspeptidase, related to hepatic enzymatic inducing effect of phenobarbital. An isolated and moderate elevation of a transaminase and / or alkaline phosphatase. Also the Phenobarbital can induce a hepatitis.

Case 10: Hematemesis of moderate abundance with epigastralgia (Case notified by Pediatric Nephrology Department on 28/02/2013)

The patient (M.M) (male), 11 years old. The starting date for taking the drug (acetylsalicylic acid, 1 tablet oral) was 26/02/2013 and it was stopped on the same day. The time of appearance of the ADRs was $4 \mathrm{~h}$. The description of the adverse effect was Hematemesis of moderate abundance with epigastralgia. The evolution of the patient was favorable.

Gastrointestinal effects Abdominal pain: Digestive hemorrhages (hematemesis, melena ...) or occult, responsible for iron deficiency anemia. These haemorrhages are all the more frequent as the dosage is higher; Gastric ulcers and perforations;

In a prospective descriptive study conducted with gastroenterologists, private and public sector of the city of Rabat, in the services surgical emergencies and the service of gastroenterology of medicine C Ibn Sina Hospital Center university of the city of Rabat, on a period of 14 months (April 2001 to May 2002). In our population, the main reason patient consultation was the digestive hemorrhage that concerned 65 patients and manifested 13 isolated hematemesis, 32 hematemesis associated with a moelena, 11 moelena, 7 haematemesis associated with anemia, 1 hematemesis associated with a moelena and Massive and 1 moelena associated with rectorragies. Epigastralgia were the second reason for consultation (45 patients), followed by the syndrome dyspeptic (14 patients). Aspirin is the most incriminated NSAID in our series (27.5\%).

\section{DISCUSSION}

This study confirms the current difficulties in recollecting drug adverse reaction notifications in practical terms in a hospital structure. The difficulty of having an adverse reaction report card complete with all the information needed to carry out a complete case-load study (chronological and semiological study and accountability score study).

In the results section, we can find cases where the information at the level of the file is missing and they have been raised in this article to insist that the field pharmacovigilance is responsible for difficulties in collecting all the information's (due to the speed of the notification, the lack of information at the time of notification by the notifier, the lack of all biological results at the time of notification, the transition of the patient from one service to another, the discharge of the patient from the hospital, etc. .)

Pharmacovigilance are all the techniques of identification, evaluation and prevention of risk of Drug adverse events, and follows a similar methodology in the child and in the adult. The "spontaneous notification" is fundamental to generating alerts that will or will not be confirmed by pharmacoepidemiological studies. ${ }^{6}$

In a spontaneous notification of an ADR, the relationship between each medicine taken by the child and the occurrence of the EIM (accountability) is established by logical reasoning that requires dialogue between the declaring clinician and the pharmacovigilance doctor.
The semiological analysis of the EIM implies a good knowledge of the clinic pediatric, and especially neonatology. Spontaneous declaration is far from as it is estimated that only 1 to $10 \%$ of serious adverse effects are reported. This underreporting is explained by a lack of time, a misunderstanding of his interest in terms of public health, the link not established by the doctor between an effect and a drug, but also by the fear of declaring an EIM after an off-label prescription.

During the study period, the most frequently reported ATC (anatomical, therapeutic and chemical) classes were vaccines, antibiotics, paracetamol, phenobarbital and valproic acid.

The most frequent adverse events were cutaneous and allergic reactions like rash, generalized eruption, erythema-papular eruption, marbling, and diffuse pruritic allergic reaction; also liver function assignments like biological hepatitis, hepatic cytolysis and Cholestasis; there were cases of adverse reactions with high fever and cases with myelosuppression and Hematemesis with epigastralgia.

The analysis of the reported cases and the age range of the children are very varied as well as the undesirable effects observed, with very severe cases and others being moderately severe. The evolution of the patients was favorable or with a medical follow-up according to the cases;

Betalactamines are probably the most widely prescribed class of antibiotics in the pediatric population, but also most often responsible for hypersensitivity reactions. The term "drug allergy" should only be used when a definite immunological mechanism is established.

From a clinical point of view, we distinguish between immediate reactions that occur within one hour after taking the drug, and non-immediate reactions whose time of onset is between one hour and several days. In an immediate reaction, we find the classic manifestations of anaphylaxis (urticaria, angioedema, rhinitis, asthma and for the most serious, anaphylactic shock). In the case of non-immediate reactions, most manifestations are manifested by a maculopapular rash. Cutaneous side effects drugs are the most frequent medical accidents notified to the pharmacovigilance centers. Events the most usual are the eruptions maculopapular (toxidermal exanthemas) and urticaria. Dermatoses bullous, erythroderma are rare. The Bullous toxidermias (fixed erythema pigmentosa) is the only eruption that is always of medicinal origin. It is marked by the sudden appearance of one or several erythematous lesions and oedematous; preferred locations are periorifical, especially in the face (lips), at the level of the genital regions and perineal. Most often incriminable drugs are sulfamethoxazole, and trimethoprim, ciprofloxacin, barbiturates.

When a medicine is allowed to be put on the market it is necessary and useful to proceed to tests on the animal to discover its toxic effect. Clinical trials focus to demonstrate the effectiveness of drugs in terms of statistical significance rather compares their benefits and adverse effects with those of drugs already existing. The duration too short clinical trials and the reduced number of patients who participate in it limit the value of their results. That's why the data clinical about the drugs before their placing on the market contain only information on adverse effects. In addition, these tests use precise doses, and patients who may be more exposed to adverse effects are usually not studied in the trials before AMM (young children, elderly people, women pregnant or lactating,.). Clinical trials so bring very informative

limited information on adverse effects and efficacy drugs in practice. It is necessary to improve the description and reporting adverse reactions in clinical trials.

\section{CONCLUSION}

The pediatric population is very sensitive to the undesirable effects of the drugs due to its physiological peculiarity and the undesirable effects can 
have detrimental effects on this population from which the importance of the pharmacovigilance and the postponement of the spontaneous notifications.

The analysis of notifications of adverse drug reactions is an important source of information and is underutilized in pediatrics. As in adults, spontaneous notification is essential to generate alerts and child-specific pharmaco-epidemiological studies are necessary and should be developed. ADR reporting to the pharmacovigilance system, in particular pediatric ADRs, should be encouraged.

\section{ACKNOWLEDGEMENT}

We thank the Colleagues in the Anti- Poison and Pharmacovigilance Centre in Rabat, all the Doctors in the Children's Hospital Committee and the Pharmacy Department of the Hospital and the Medical Professionals for his support on the project.

\section{CONFLICT OF INTEREST}

The authors declare no conflict of interest.

\section{ABBREVIATIONS}

ADRs : adverse drug reactions; HER: Children's Hospital of Rabat; ANSM: The Agence Nationale de sécurité du Médicament et des produits de santé; CAPM: Poison Control and Pharmacovigilance center of Rabat.

\section{SUMMARY}

Many drugs are given in pediatrics according to off-label prescriptions which exposes the child to a increased risk of errors. This practice is due to a lack of evaluation of drugs nevertheless essential because of the pharmacological peculiarities of the child.

\section{REFERENCES}

1. Bégaud B, Evreux JC, Jouglard J, Lagier G. Imputabilité des effets inattendus ou toxiques des médicaments. Actualisation de la méthode utilisée en France. Thérapie . 1985;40(2):111-8.

2. Yee D, Valiquette C, Pelletier M, Parisien I, Rocher I, Menzies D. Incidence of serious side effects from first-line antituberculosis drugs among patients treated for active tuberculosis. Am J Respir Crit Care Med. 2003;167(11):1472-7.

3. Brogden RN, Rifabutin FA. A review of its antimicrobial activity, pharmacokinetic properties and therapeutic efficacy. Drugs. 1994;47(6):983-1009.

4. Thompson NP, Caplin ME, Hamilton MI, Gillespie SH, Clarke SW, Burroughs AK, et al. Anti-tuberculosis medication and the liver: Dangers and recommendations in management. Eur Respir J. 1995;8(8):1384-8.

5. Harr T, French LE. Toxic epidermal necrolysis and Stevens-Johnson syndrome. Orphanet J Rare Dis. 2010;5(1):39.

6. Autret-Leca E, Marchand MS, Cissoko H, Beau-Salinas F, Jonville-Béra AP. Pharmacovigilance en pédiatrie. Arch Pédiatrie. 2012;19(8):848-55.

Article History: Submission Date : 02-04-2018; Revised Date : 15-05-2018; Acceptance Date : 08-06-2018.

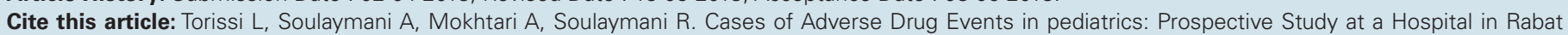
(Morocco). J Young Pharm. 2018;10(3):362-6. 